\title{
Simply supported composite hypar shells under free vibration-some observations
}

\begin{abstract}
A general finite element procedure is presented to model the hypar shells using eight-noded curved quadratic isoparametric elements with five degrees of freedom per node including two inplane displacements and one transverse displacement and two rotations. Problems of twisted cantilever plate, which have structural resemblance with skewed hypar shell, are solved using the present approach and the results are compared with published ones. Having established the exactitude of the present formulation, numerical experiments with simply supported skewed composite hypar shells are conducted for four different types of laminations including four layered symmetric and antisymmetric cross and angle ply laminates. The first four natural frequencies are presented in tabular forms and are studied critically and a set of meaningful conclusions are derived.
\end{abstract}

Keywords: hypar shell, composite, natural frequency
Volume 2 Issue 4 - 2018

\section{Sarmila Sahoo}

Heritage Institute of Technology, India

Correspondence: Sarmila Sahoo, Department of Civil Engineering, Heritage Institute of Technology, Kolkata- 700 107, India, Email sarmila.sahoo@gmail.com

Received: November 27, 2017 | Published: August 28, 2018

\section{Introduction}

Hyperbolic paraboloid shell bounded by straight lines (commonly known as hypar shell) is a good choice as roofing unit to civil engineers due to its aesthetic beauty and capability to allow entry of north light. In this age of advanced materials composite skewed hypars define a rich area of research. Schwarte ${ }^{1}$ worked on free vibration of isotropic rhombic hypar shell and the twisted plates which have structural resemblance with hypar shells received attention from several authors like Kielb, ${ }^{2}$ Seshu \& Ramamurti ${ }^{3}$ and others. Chakravorty et al., ${ }^{4}$ in 1998 reported natural frequency and forced vibration response of corner point supported skewed hypar shell.

Thus it is evident that most of the work on hypar shells deals with fundamental frequency and frequency for higher modes received limited attention only. Moreover the effect of neglecting tangential and /or rotary inertia on the natural frequencies of hypar shell has not received any attention. The first four natural frequency of simply supported composite hypar shell and the effect of neglecting tangential and / or rotary inertia on them is presented.

\section{Mathematical formulation}

An eight-noded curved quadratic isoparametric finite element is used for hypar shell analysis. The five degrees of freedom taken into consideration at each node are $\mathrm{u}, \mathrm{v}, \mathrm{w}, \alpha, \beta$. The strain-displacement relations on the basis of improved first order approximation theory for thin shell are established which was provided by solution of benchmark problems reported elsewhere ${ }^{5}$ and are established as

$$
\begin{gathered}
\left\{\begin{array}{lllll}
\varepsilon_{x} & \varepsilon_{y} & \gamma_{x y} & \gamma_{x z} & \gamma_{y z}
\end{array}\right\}^{T}=\left\{\begin{array}{lllll}
\varepsilon_{x}^{0} & \varepsilon_{y}^{0} & \gamma_{x y}^{0} & \gamma_{x z}^{0} & \gamma_{y z}^{0}
\end{array}\right\}^{T} \\
+z\left\{\begin{array}{llllll}
k_{x} & k_{y} & k_{x y} & k_{x z} & k_{y z}
\end{array}\right\}^{T}
\end{gathered}
$$

Where the first vector is the mid-surface strain for a hypar shell and the second vector is the curvature. These are given, respectively, by

$$
\left\{\begin{array}{c}
\varepsilon_{x}^{0} \\
\varepsilon_{y}^{0} \\
\gamma_{x y}^{0} \\
\gamma_{x z}^{0} \\
\gamma_{y z}^{0}
\end{array}\right\}=\left\{\begin{array}{c}
\partial u / \partial x \\
\partial v / \partial y \\
\partial u / \partial y+\partial v / \partial x-2 w / R_{x y} \\
\alpha+\partial w / \partial x \\
\beta+\partial w / \partial y
\end{array}\right\},
$$

$$
\left\{\begin{array}{c}
k_{x} \\
k_{y} \\
k_{x y} \\
k_{x z} \\
k_{y z}
\end{array}\right\}=\left\{\begin{array}{c}
\partial \alpha / \partial x \\
\partial \beta / \partial y \\
\partial \alpha / \partial y+\partial \beta / \partial x \\
0 \\
0
\end{array}\right\}
$$

A laminated composite hypar shell of uniform thickness $h$ and twist radius of curvature Rxy is considered. Keeping the total thickness same, the thickness may consist of any number of thin laminae each of which may be arbitrarily oriented at an angle $\theta$ with reference to the $\mathrm{x}$-axis of the co-ordinate system. The constitutive equations for the shell are given by

$$
\begin{aligned}
& \{F\}=[D]\{\varepsilon\} \\
& \{F\}=\left\{\begin{array}{llllllll}
N_{x} & N_{y} & N_{x y} & M_{x} & M_{y} & M_{x y} & Q_{x} & Q_{y}
\end{array}\right\}^{T}, \\
& {[D]=\left[\begin{array}{lll}
{[A]} & {[B]} & {[0]} \\
{[B]} & {[D]} & {[0]} \\
{[0]} & {[0]} & {[S]}
\end{array}\right],} \\
& \{\varepsilon\}=\left\{\begin{array}{llllllll}
\varepsilon_{x}^{0} & \varepsilon_{y}^{0} & \gamma_{x y}^{0} & k_{x} & k_{y} & k_{x y} & \gamma_{x z}^{0} & \gamma_{y z}^{0}
\end{array}\right\}^{T} .
\end{aligned}
$$

The stiffness coefficients are defined as

$$
\begin{gathered}
A_{i j}=\sum_{k=1}^{n p}\left(Q_{i j}\right)_{k}\left(z_{k}-z_{k-1}\right) ; \\
B_{i j}=\frac{1}{2} \sum_{k=1}^{n p}\left(Q_{i j}\right)_{k}\left(z_{k}^{2}-z_{k-1}^{2}\right) ; \\
D_{i j}=\frac{1}{3} \sum_{k=1}^{n p}\left(Q_{i j}\right)_{k}\left(z_{k}^{3}-z_{k-1}^{3}\right) \quad \mathrm{i}, \mathrm{j}=1,2,6 ; \\
S_{i j}=\sum_{k=1}^{n p} F_{i} F_{j}\left(G_{i j}\right)_{k}\left(z_{k}-z_{k-1}\right) \quad \mathrm{i}, \mathrm{j}=1,2 ;
\end{gathered}
$$


Where $\mathrm{Q}_{\mathrm{ij}}$ are elements of the off-axis elastic constant matrix which are derived from appropriate transformation of the on-axis matrix. $F_{i}$ and $\mathrm{F}_{\mathrm{j}}$ are shear correction factors presently taken as unity. The terms of the on-axis matrix depend on the elastic moduli and Poisson's ratio of the material. The element stiffness matrix and the load vector are derived through the routine steps of finite element formulation employing numerical integration. The element stiffness matrix and the element load vectors are assembled to get the global matrices. The basic equation of statics is solved by Gaussian elimination algorithm. The element matrices are assembled after performing appropriate transformations due to the curved shell surface to obtain the respective global matrices $[\mathrm{K}]$ and $[\mathrm{M}]$. The free vibration analysis involves determination of natural frequencies from the condition

$$
\left|[K]-\omega^{2}[M]\right|=0
$$

This is a generalized eigen value problem and is solved by the subspace iteration algorithm.

\section{Numerical examples}

Cantilever twisted plates are solved (done earlier by Qatu \& Leissa $^{6}$ ) to obtain the non-dimensional fundamental frequencies and the present results are compared with the published ones (Table 1). Additional problems of skewed hypar shells (Figure 1) are also solved considering different stacking sequences and simply supported boundary. The first four natural frequencies are obtained and presented in the form of table using the non-dimensional parameter $\omega$. The values in parentheses indicates the percentage deviations of the frequencies when neglecting tangential and/or rotary inertia from those obtained by considering all d.o.f.s with the latter as the base. In all the cases only the converged results are presented in Table 2 . The fundamental frequencies are taken to have converged for particular finite element grid, if further refinement of the grid does not improve that result by more than one percent. With this criterion an $8 \times 8$ mesh is found to be appropriate for all the problems taken up here.

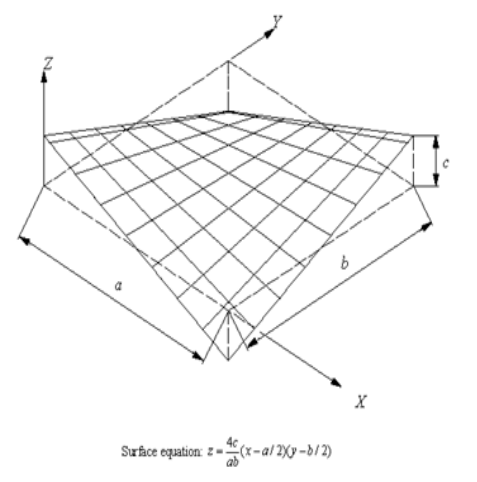

Figure I Surface of a skewed hypar shell.

Table I Non-dimensional natural frequencies $\bar{\omega}\left[=\omega a^{2}\left(\rho / E_{11} h^{2}\right)^{1 / 2}\right]$ for three layer graphite epoxy twisted plates, $[\theta /-\theta / \theta]$ laminates

\begin{tabular}{lllllllll}
\hline & $\theta(\delta \varepsilon \gamma \rho \varepsilon \varepsilon)$ & 0 & 15 & 30 & 45 & 60 & 75 & 90 \\
& Qatu and Leissa[6] & 1.0035 & 0.9296 & 0.7465 & 0.5286 & 0.3545 & 0.2723 & 0.2555 \\
$\varphi=150$ & Present FEM & 0.9989 & 0.9258 & 0.7443 & 0.5278 & 0.3541 & 0.272 & 0.2551 \\
\hline
\end{tabular}

$\mathrm{a} / \mathrm{b}=\mathrm{I}, \mathrm{a} / \mathrm{h}=100 ; \mathrm{EI} \mathrm{I}=\mathrm{I} 38 \mathrm{GPa}, \mathrm{E} 22=8.96 \mathrm{GPa}, \mathrm{GI} 2=7 . \mathrm{I} \mathrm{GPa}, v_{12}=0.3, \mathrm{~h}$ - shell thickness

Table 2 First four natural frequencies $(\mathrm{Hz})$ for laminated composite hypar shells $(a / b=I)$ with different stacking sequences

\begin{tabular}{|c|c|c|c|c|}
\hline \multirow[t]{2}{*}{$\begin{array}{l}\text { Lamination } \\
\text { (degree) }\end{array}$} & $\begin{array}{l}\text { Frequencies } \\
\text { with all d.o.f. }\end{array}$ & $\begin{array}{l}\text { Frequencies } \\
\text { without } \\
\text { tangential } \\
\text { inertia }\end{array}$ & $\begin{array}{l}\text { Frequencies } \\
\text { without rotary } \\
\text { inertia }\end{array}$ & $\begin{array}{l}\text { Frequencies } \\
\text { without both } \\
\text { tangential and } \\
\text { rotary inertia }\end{array}$ \\
\hline & (A) & (B) & (C) & (D) \\
\hline \multirow[t]{8}{*}{$0 / 90 / 90 / 0$} & 5.12303 & 5.56508 & 5.12326 & 5.56538 \\
\hline & & -8.545 & -0.004 & -8.635 \\
\hline & 5.431 & 5.90314 & 5.43127 & 5.90348 \\
\hline & & -8.693 & -0.005 & -8.67 \\
\hline & $6.7700 \mathrm{I}$ & 7.07466 & 6.77061 & 7.07534 \\
\hline & & -4.5 & -0.009 & $-4.5 I$ \\
\hline & 9.26257 & 9.71075 & 9.26408 & $9.7 \mid 251$ \\
\hline & & -4.51 & -0.016 & -4.858 \\
\hline \multirow[t]{6}{*}{$0 / 90 / 0 / 90$} & 5.15533 & 5.59977 & 5.15557 & 5.60008 \\
\hline & & -8.622 & -0.005 & -8.627 \\
\hline & 5.20721 & 5.65169 & 5.20746 & 5.652 \\
\hline & & -8.536 & -0.005 & -8.542 \\
\hline & 7.99776 & 8.3755 & 7.99848 & 8.37632 \\
\hline & & -4.723 & -0.009 & -4.733 \\
\hline
\end{tabular}


Table Continued

\begin{tabular}{|c|c|c|c|c|}
\hline \multirow[t]{3}{*}{$\begin{array}{l}\text { Lamination } \\
\text { (degree) }\end{array}$} & $\begin{array}{l}\text { Frequencies } \\
\text { with all d.o.f. } \\
\text { (A) }\end{array}$ & $\begin{array}{l}\text { Frequencies } \\
\text { without } \\
\text { tangential } \\
\text { inertia } \\
\text { (B) }\end{array}$ & $\begin{array}{l}\text { Frequencies } \\
\text { without rotary } \\
\text { inertia } \\
\text { (C) }\end{array}$ & $\begin{array}{l}\text { Frequencies } \\
\text { without both } \\
\text { tangential and } \\
\text { rotary inertia } \\
\text { (D) }\end{array}$ \\
\hline & 8.037 & 8.40747 & 8.03767 & 8.40827 \\
\hline & & -4.61 & -0.008 & -4.62 \\
\hline \multirow[t]{8}{*}{$45 /-45 /-45 / 45$} & 5.78153 & 6.32587 & 5.78182 & 6.32624 \\
\hline & & -9.415 & -0.005 & -9.422 \\
\hline & 6.06977 & 6.65609 & 6.07006 & 6.65648 \\
\hline & & -9.66 & -0.005 & -9.666 \\
\hline & 8.03849 & 8.45823 & 8.03926 & 8.45912 \\
\hline & & -5.222 & -0.009 & -5.233 \\
\hline & 10.9619 & 11.5318 & 10.9631 & 11.5332 \\
\hline & & -5.199 & -0.011 & -5.212 \\
\hline \multirow[t]{8}{*}{$45 /-45 / 45 /-45$} & 6.0927 & 6.66584 & 6.09301 & 6.66624 \\
\hline & & -9.407 & -0.005 & -9.414 \\
\hline & 6.12258 & 6.69458 & 6.12289 & 6.69498 \\
\hline & & $(9.342))$ & -0.005 & -9.348 \\
\hline & 8.06525 & 8.47611 & 8.06601 & 8.477 \\
\hline & & -5.094 & -0.009 & -5.105 \\
\hline & 11.3798 & 11.9632 & $|1.38|$ & 11.9646 \\
\hline & & -5.127 & -0.011 & -5.139 \\
\hline
\end{tabular}

$\mathrm{a} / \mathrm{b}=\mathrm{I}, \mathrm{a} / \mathrm{h}=\mathrm{I00}, \mathrm{c} / \mathrm{a}=0.2 ; \mathrm{EI} \mathrm{I}=25 \mathrm{E} 22, \mathrm{GI} 2=\mathrm{GI} 3=0.5 \mathrm{E} 22, \mathrm{G} 23=0.2 \mathrm{E} 22, \mathrm{v}_{12}=\mathrm{V}_{21}=0.25$

(Values in the parentheses are the percentage deviations of values in columns

\section{Results and discussions}

From Table 1 it is found that the fundamental frequencies of cantilever twisted plates obtained by Qatu \& Leissa ${ }^{6}$ compare well with the present results. So the correctness of the present approach incorporating the effect of twist of curvature in the formulation is established.

It is seen from Table 2 that the frequencies increase when the tangential and or rotary inertia are neglected. The increased values of the frequencies are quite justified because the elimination of some terms from the mass matrix leads to stiffer modeling of the shells. It is also observed that in almost all the cases the percentage deviations of the frequencies when neglecting tangential inertia and rotary inertia separately can be added up to obtain the percentage deviation for the case when both tangential inertia and rotary inertia are neglected. Thus the principle of superposition seems to be applicable. However, in all the problems the above effects are negligible. If the tangential inertia terms are neglected there is an over estimation of natural frequencies no doubt but such deviations are acceptable from engineering point of view being less than $10 \%$. This means almost correct natural frequencies may be obtained with much less computational effort. Physically, the results imply that the major part of kinetic energy associated with shell vibration is due to transverse displacement.

An in-depth study of the percentage deviation of the frequencies caused due to negligence of tangential and/ or rotary inertia further reveals that the effects of neglecting tangential inertia are much
$(B),(C)$ and $(D)$ with respect to those in column (A).).

more pronounced than those of neglecting rotary inertia. Therefore, in hypar shells the contribution of the tangential movements is more conspicuous than that of the rotary movements in the total kinetic energy of vibration. Again from Table 2 it is also found that the percentage deviation of natural frequency is more in angle ply shells than that in cross ply shells. But comparing the percentage deviation of anti-symmetric and symmetric cross and angle ply shells no unified conclusion can be drawn.

\section{Conclusion}

The present finite element approach is capable of modelling free vibration problem of shells with twist of curvature. The effect of neglecting tangential and rotary inertia to obtain the natural frequencies is negligible. This means almost correct natural frequencies may be obtained with much less computational effort. Physically, the results imply that the major part of kinetic energy associated with shell vibration is due to transverse displacement.

In hypar shells the contribution of the tangential movements is more conspicuous than that of the rotary movements in the total kinetic energy of vibration. The percentage deviation of natural frequency is more in angle ply shells than that in cross ply shells. But comparing the percentage deviations of anti-symmetric and symmetric cross and angle ply shells no unified conclusion comes out.

\section{Acknowledgments}

None. 


\section{Conflict of interest}

Author declares there is no conflict of interest.

\section{References}

1. Schwarte J. Vibrations of corner point supported rhombic hypar-shells. $J$ Sound Vibr. 1994;175(1):105-114.

2. Kielb RE, Leissa AW, Macbain JC, et al. Vibrations of twisted cantilever plates-a comparison of theoretical results. Int $J$ for Num Methods in Engng. 1985;21(8):1365-1380.
3. Seshu P, Ramamurti V. Vibrations of twisted composite plates. $J$ Aeronautical Soc India. 1989;41:65-70.

4. Chakravorty D, Bandyopadhyay JN, Sinha PK, et al. Applications of FEM on free and forced vibrations of laminated shells. ASCE, J Engng Mech. 1998;124(1):1-8.

5. Sahoo S, Chakravorty D. Finite element bending behaviour of composite hyperbolic paraboloidal shells with various edge conditions. J Strain Analysis for Engng Design. 2004;39(5):499-513.

6. Qatu MS, Leissa AW. Vibration studies for laminated composite twisted cantilever plates. Int J Mech Sc. 1991;33(11):927-940. 